\title{
Cancer Might be Manifested by Short Breathing
}

\author{
Ramesh Kumar Sharma* \\ Scientific Writer on Food and Environment Issues, India
}

ISSN: 2637-773X

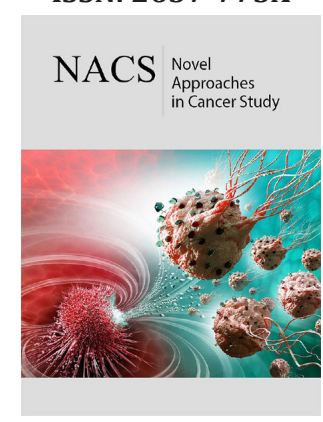

*Corresponding author: Ramesh Kumar Sharma, Scientific Writer on Food and Environment Issues, India

Submission: 阱 April 24, 2019

Published: 海 May 21, 2019

Volume 2 - Issue 5

How to cite this article:Ramesh $\mathrm{K} \mathrm{S}$. Cancer Might be Manifested by Short Breathing. Nov Appro in Can Study. 2(5). NACS.000547.2019.

DOI: 10.31031/NACS.2019.02.000547

Copyright@ Ramesh Kumar Sharma, This article is distributed under the terms of the Creative Commons Attribution 4.0 International License, which permits unrestricted use and redistribution provided that the original author and source are credited.

\section{Opinion}

It is normally believed that the most causes of short breathing are due to heart and lung conditions, say asthma or allergic reactions or heart attack or low blood pressure or anemia or pneumonia or carbon monoxide poisoning or upper airway obstruction or blood clot in lungs; but chronic shortness of breath is most often due to asthma, chronic obstructive pulmonary disease, obesity, and lung diseases including the cancer of organ [1]. At this point the question arises, whether the chronic dyspnea (shortness of breath) is the symptom of lung cancer or might be its possible cause. However, it is found that people with advanced cancer (not only lung cancer) often get shortness of breath or dyspnea [2]. Therefore, the raised question is more generalized; whether chronic dyspnea is a cause or just a symptom of cancer. In other words, question arises, whether the dyspnea is the result of carcinogenic conditions in or cancer is caused by dyspnea state of the body. In 1986, Desigan et al. [3] tried to correlate progressive shortness of breath with manifestation of occult gastric cancer and stated that progressive shortness of breath may be the first or only manifestation of occult gastric cancer caused by either lymphangitic carcinomatosis or microscopic tumor emboli to the lungs. In this context, a further question arises, whether Desigan [3] statement might be generalized for various carcinogenic conditions in body. That means, might it be a true statement: progressive shortness of breath is the major manifestation of cancer or cancer is often manifested by progressive shortness of breath.

There appears a reason for not accepting dyspnea as a manifestation of all types of cancers. Dyspnea is prevalent in $50 \%$ to $60 \%$ of patients with advanced cancer generally, and in up to $74 \%$ of patients with lung cancer; prevalence increases during the last six weeks of life and can cause significant psychological emotion distress [4]. Still the hypothesis 'cancer is often manifested by progressive shortness of breath' might numerically be presented as: cancer is generally manifested by progressive shortness of breath in around 55\% (50-60\%) of cancer patients and up to $74 \%$ of lung cancer patients. Whether these large number of cancer cases are manifested by short breathing or breathing disorders long before a tumor is formed in body, is the question that originates in this context. However, the hypothesis testing perhaps is a difficult task due to unavailability of early stage (before formation of tumor) breathing complications of a cancer patient. Normally a person hardly recognizes breathing complication, whether due to high particulate matter air environment or suffering from sinusitis or any other reason, when the situation becomes chronically severe. The question, therefore, remains as such: the cancer is manifested by progressive shortness of breath or dyspnea is just its symptom.

The trend set of dyspnea associated cancer cases, increasing or decreasing with time, might indicate something in this context. According to Medline search of the literature published from 1966 to 1999, dyspnea occurred in 21-78.6\% patients of advanced cancer (moderate to severe $10-63 \%$ of the patients) in this period depending upon the types of cancers (needless to say maximum in $78.6 \%$ of lung cancer patients) [5]. On the other hand, according to a 2017-report, dyspnea is prevalent in $50 \%$ to $60 \%$ of patients with advanced cancer generally, and in up to $74 \%$ of patients with lung cancer [4]. A comparison of these two reports indicates that prevalence of dyspnea is reduced in the cases of more specific dyspnea cancers $(78.6 \%$ to $74 \%$ lung cancer; $63 \%$ to $60 \%$ upper limit of dyspnea prevalence in other cancers) with passage of time. But limit of prevalence of dyspnea in cancers other than lung 
cancer is narrowed and lower limit is increased from 21\% to $50 \%$ with time. Of course, the upper limit of dyspnea prevalence in cancer cases might be reduced with passage of time due to management of dyspnea in advanced cancer patients which includes oxygen therapy, use of benzodiazepines etc. [5]. But general increase of dyspnea patients in different cancer cases with time is perhaps indicative of polluting atmosphere with increasing particulate matter; making people increasingly suffer from sinusitis and later on dyspnea cancer. If it is true, the cancer might be manifested by short breathing; and control of particulate matter in atmosphere which seems possible by dense forestation, appears to be the prime requirement for cancer control..

\section{References}

1. CHEST Foundation (The philanthropic arm of the American College of Chest Physicians). Shortness of breath: symptoms, causes and risk factors. (C) 2019: American Lung Association.

2. The cancer council of Australia. Shortness of breath. www.cancercouncil. com.au

3. Desigan G, Wang M, Wofford B, Dunn GD, Vaughan S(1986) Occult gastric cancer manifested by progressive shortness of breath in a young adult. South Med J 79 (9):1173-1176.

4. Jenkins K(2017) Dyspnea in advanced cancer: Dying long before death. Medscape.

5. Ripamonti C(1999) Management of dyspnea in advanced cancer patients. Support Care Cancer 7(4): 233-243. 